\title{
Establishing moral bearings: ethics and expatriate health care professionals in humanitarian work
}

\author{
Matthew R. Hunt, PhD Post-Doctoral Fellow, Centre for Research on Ethics, \\ University of Montreal and Post-Doctoral Fellow, Department of Clinical \\ Epidemiology and Biostatistics, McMaster University, Canada
}

Expatriate health care professionals frequently participate in international responses to natural disasters and humanitarian emergencies. This field of practice presents important clinical, logistical and ethical challenges for clinicians. This paper considers the ethics of health care practice in humanitarian contexts. It examines features that contribute to forming the moral landscape of humanitarian work, and discusses normative guidelines and approaches that are relevant for this work. These tools and frameworks provide important ethics resources for humanitarian settings. Finally, it elaborates a set of questions that can aid health care professionals as they analyse ethical issues that they experience in the field. The proposed process can assist clinicians as they seek to establish their moral bearings in situations of ethical complexity and uncertainty. Identifying and developing ethics resources and vocabulary for clinical practice in humanitarian work will help health care professionals provide ethically sound care to patients and communities.

Keywords: decision-making, ethics, health care professionals, humanitarian emergencies, natural disasters, non-governmental organisations

\section{Introduction}

Expatriate health care professionals (HCPs) frequently participate in international responses to natural disasters and humanitarian emergencies. ${ }^{\mathrm{I}}$ This field of practice presents important clinical, logistical and ethical challenges for clinicians. Health care and related interventions often are complicated by unstable security situations, a lack of human and material resources, political obstacles to care delivery and rapidly changing health needs of the population (Banatvala and Zwi, 200o). HCPs who travel from a developed nation to a low-resource setting where a humanitarian crisis has occurred also experience a shift of professional, social, cultural and regulatory environments. This setting is substantially different to their 'ordinary' practice environment. Human rights, public health, medicine and ethics intersect in different ways during health care practice in humanitarian contexts (Mann, I997). The moral dimension of humanitarian work is rendered more complex by the international and trans-cultural nature of this work.

In the past two decades there has been considerable discussion within the humanitarian community, and by academics, regarding the ethics of humanitarian response 
(Slim, I997; Moore, I998; Terry, 2002; IFRC, 2003). One aspect of this debate considers the types of dilemmas that humanitarian organisations navigate during programme development and decision-making. A central focus has been on evaluating how humanitarian interventions designed to assist populations in need sometimes result in unintended harm of beneficiaries (de Waal, I998; Anderson, 2000). For example, the presence of humanitarian agencies may inadvertently contribute to the perpetuation of conflict or aid may be co-opted to promote the war aims of belligerents (Horton, 2008). Other aspects of this analysis include the place of fundamental humanitarian principles in guiding organisational decisions during relief work (Leader, I998), interactions between militaries and humanitarian organisations (Wheeler and Harmer, 2006), organisational approaches to resource allocation (Fuller, 2006; Hurst, Mezger and Mauron, 2009), the promotion of accountability (Humanitarian Accountability Partnership-International, 2007) and the influence of foreign policy objectives on relief work (Frangonikolopoulus, 2005). This discussion has led to a number of international initiatives designed to provide standards of best practice for humanitarian actors, evaluate outcomes, and increase the accountability of aid organisations (Sphere Project, 2004; Humanitarian Accountability PartnershipInternational, 2007).

While there has been extensive discussion of ethics at the level of organisations and programmes, there has been less examination of ethical issues experienced by expatriate HCPs deployed on humanitarian missions. HCPs involved in humanitarian work are frequently confronted by morally complex situations. In a qualitative study of how health professionals experience ethics in humanitarian assistance and development work, this author identified the following themes: I) tension between respecting local customs and values, and acting in ways that are consistent with one's core moral convictions; 2) barriers to the provision of adequate care; 3 ) divergent understandings and experiences of health and illness; 4) questions of identity as a professional, humanitarian and moral person; and 5) issues of trust and distrust (Hunt, 2008). Other key aspects of the moral experience of HCPs in humanitarian work include clarifying motivations and expectations, the relationality of humanitarian action, attending to steep imbalances of power, acknowledging and confronting limits to what can be accomplished, and recognising how organisational structures shape everyday moral experience (Hunt, 2009).

Many ethical issues in humanitarian health practice are resolved by HCPs on a routine basis. Other issues present significant challenges and can lead to moral uncertainty or distress. This paper seeks to identify resources to assist HCPs in analysing and responding to ethical issues that arise in health care practice in humanitarian settings. It assesses some of the particular features of humanitarian assistance that contribute to the ethical complexity of this work. ${ }^{2}$ Next it briefly surveys relevant ethics resources and frameworks, including resources specific to humanitarian settings and resources related to health care ethics more generally. Finally, it proposes a set of questions to support reflection and discussion of ethically complex issues in health care practice during humanitarian crises. 


\section{Moral landscape of humanitarian work}

Various features contribute to the complexity of ethical issues for expatriate HCPs in humanitarian work. This paper highlights six key features of this field of practice. First, humanitarian work is characterised by contexts where available resources of personnel, supplies and health infrastructure are insufficient to meet the needs of patients and populations. These limitations often are severe, and are not restricted to health care. There may be inadequate food, sanitation, shelter, potable water and security for affected communities. In some settings, populations have been displaced from their homes and may be living in temporary camps for refugees or internally displaced persons. Expatriate HCPs may struggle to adapt to settings of acute resource scarcity and the frequent need to allocate limited resources (Michael and Zwi, 2002). They will be challenged by the need to contextualise their clinical knowledge and training when there are severe constraints on available diagnostic, referral and intervention options. In an acute emergency, the level of achievable care probably will be much lower than what is familiar to many expatriate HCPs. In such settings, triage of resources and services may be commonplace. HCPs need to adapt their clinical approach as a result.

Second, in contexts of resource scarcity, increased instability, and widespread health needs, population health concerns abound. Public health experts, as well as water and sanitation engineers, are key members of relief operations and respond to many public health needs. Expatriate HCPs in humanitarian work also need to maintain a heightened attentiveness to population-based concerns (Brennan and Nandy, 200I). As Hakewill (I997, p. 62I) notes, HCPs must 'reorient their thinking from a purely clinical approach to a constant preoccupation with public health interventions'. Some HCPs who do not have a background in public health may be challenged as they move between addressing the needs of individual patients in their care, and the concerns of the broader population (Hunt, 2008). In discussing ethical issues associated with implementation of antiretroviral programmes by a nongovernmental organisation (NGO) in a low resource setting, Benatar (2006, p. 323) describes how there is the 'potential for conflict among several principles of public health ethics and between the ethics of individual care and public health ethics'. These conflicts may result in uncertainty, particularly for HCPs trained within health care systems where public health receives less emphasis than the delivery of care to individual patients.

A third feature of health care practice in humanitarian crises relates to work styles and human resource practices in humanitarian agencies (People in Aid, 2003). In humanitarian settings the volume and urgency of needs of the local population are elevated. HCPs often work extremely long shifts and have limited opportunity to pause and reflect on their work. They may experience overwork and exhaustion. This situation will be most acute during the initial response to a disaster or humanitarian emergency. However, there may be a tendency to put in long hours and maintain this intensity even when the acute phase has passed. Hilhorst and Schmiemann (2002, p. 497) note in their study of the organisational culture of Médecins Sans 
Frontières-Holland how some expatriate workers 'display an emergency work style even in non-emergency situations'. Working at such a pace may limit opportunities to explore ethically complex issues while in the field.

The high rate of expatriate staff turnover that frequently exists within humanitarian organisations has implications for organisational memory, project continuity and the development of local expertise (Loquercio, Hammersley and Emmens, 2006). Without careful planning to mitigate the impact of frequent staffing changes there may be a inclination to 'reinvent the wheel'. Rapid turnover also can be an impediment to creating and maintaining trusting and collaborative relationships with local actors. Developing and expanding the responsibilities and capacities of national staff members will help to address this concern, and contribute to enhancing local capacity.

Fourth, important differences exist between cultural frameworks in how health, wellness, disease and disability are understood and experienced (Kleinman, I980; Shweder et al., 1997). Eliciting and responding to the cultural values and needs of patients is a key aspect of clinical care. However, there are important barriers to achieving this goal in humanitarian settings. Individual HCPs bring with them their own cultural expectations and preconceptions of health and health care (Parfitt, I999). Language barriers also are common (Bjerneld et al., 2004). In this context there will be less that HCPs can take for granted and tacit knowledge of how health care is understood and experienced will be called into question (Crigger and Holcomb, 2007). Expatriate HCPs will need to consider how they address local cultural and spiritual understandings of health and illness, respond to local cultural practices, and relate to traditional health practitioners.

Fifth, imbalances of power are inherent in health care interactions. Such power differentials are amplified in humanitarian settings. Humanitarian relationships are necessarily very unequal ones. On one side are organisations and individuals that choose to work in a particular setting of need and vulnerability. They have resources at their disposal that they can give to those in need. In contrast, the affected population often is in desperate need of assistance, has few real options, and may be dependent on outside assistance for its survival. Asymmetries of power are an important consideration in health care practice in any setting; the need to account for power relations is heightened in global health contexts (Tong, 200I).

Sixth, expatriate HCPs in humanitarian work enter a field where there is less regulatory oversight and professional accountability than in their home country. Areas of broad professional and social consensus will be less certain. There may be less guidance for determining the boundaries of ethically sound practice. This will be balanced by the degree to which organisations provide detailed policies and relevant support to assist HCPs by decreasing the ambiguity of expectations and care parameters. Likewise, international initiatives to develop standards of practice and codes of conduct can assist HCPs in the field. Several of these tools are discussed in the following section. 


\section{Ethical frameworks and normative guidelines}

Humanitarian action is a moral activity grounded in an ethic of assistance to those in need (Alkire and Chen, 2004). The humanitarian ethic is linked to ideas of charity and equity. ${ }^{3}$ There remains a pressing need to think through what other ethical frameworks and approaches can promote and support moral reflection and decisionmaking as practitioners seek to act on their humanitarian ethic. What principles and values should guide the deliberations and actions of expatriate HCPs engaged in humanitarian work? International guidelines on health care ethics (such as WMA, 2006a), codes of practice for humanitarian workers (such as IFRC, I994), professional moral norms from their home country, local cultural values and moral understandings, legal and ethical parameters of health care practice in the nation where the crisis has occurred, organisational policies, and principles of international humanitarian law all provide a vantage point from which to analyse these issues. In this context, no single perspective or framework will be sufficient for resolving all ethical questions that arise in the field. Ethics analysis that critically engages relevant features of the local context (Hoffmaster, 1994) and draws on a variety of ethics resources will assist HCPs in addressing challenging issues.

\section{International ethics guidelines for health care practice}

A number of international agencies offer guidelines to aid HCPs in assessing the ethics of their work (ICN, 2006; WMA, 2006a). Some of the most important of these guidance documents are those produced by the World Medical Association (WMA), including the International Code of Medical Ethics (WMA, 2006a) and the Statement on Medical Ethics in the Event of Disasters (WMA, 2006b). The International Code of Medical Ethics asserts that doctors worldwide are duty-bound to provide technically competent care, treat patients with compassion, and respect human dignity. These guidelines articulate parameters of practice to orient the actions of professionals in diverse settings.

\section{Humanitarian guidelines}

As well as codes of ethics developed for the purposes of particular NGOs, there are several inter-agency guidance documents that aim to orient practitioners in humanitarian work. Two of these documents are The Humanitarian Charter and Minimum Standards in Disaster Response of the Sphere Project (2004) and The Code of Conduct for the International Red Cross and Red Crescent Movement and NGOs in Disaster Relief of the International Federation of Red Cross and Red Crescent Societies (IFRC, I994).

The Geneva-based Sphere Project was created in 1997 by a coalition of NGOs that sought to establish minimum standards for the delivery of humanitarian aid, as well as to encourage the humanitarian community to abide by fundamental humanitarian principles. A primary focus of The Humanitarian Charter is promotion of the 'right to life with dignity' (Sphere Project, 2004). This right is expanded on to include the right to life, the right to an adequate standard of living, and the right not to be 
subjected to cruel, inhuman or degrading treatment or punishment. The importance of promoting the dignity of aid recipients should give practitioners pause for reflection. For example, in an effort to promote patient dignity HCPs might limit access to hospitalised patients by photo-journalists after a natural disaster (Bhan, 2005). Although much of the work of the Sphere Project focuses on operational standards there is an underlying concern with the ethical treatment of affected populations and individuals in receipt of humanitarian assistance.

Also relevant to the context of health care in humanitarian work is The Code of Conduct for the International Red Cross and Red Crescent Movement and NGOs in Disaster Relief (IFRC, I994). This code aims to encourage high standards of behaviour and integrity among humanitarian actors. A number of considerations are addressed, including the right to assistance, the principles of independence, proportionality and impartiality, the responsibility to respect local culture and customs, build local capacity, address root vulnerabilities and be accountable, and the imperative of promoting the dignity of aid recipients. The code thus identifies guiding principles that signatory organisations and their staff aspire to uphold, without addressing operational questions of how they should act on them.

\section{Professional moral norms}

Expatriate HCPs who participate in humanitarian action typically are members of professional organisations in their home country. These organisations have standards of ethics and professional practice, often articulated in codes and value statements, that members are required to maintain. Professional moral norms are an essential feature of the professional identity of HCPs. Clinicians rely on these parameters of practice that are grounded in local legal requirements, as well as on social and professional consensus, to help determine ethically defensible actions while carrying out their professional responsibilities. Such codes are unlikely to be sufficient for analysing ethical challenges facing HCPs in humanitarian settings where local expectations, legal standards, clinical realities, and norms of practice may be significantly different to the context of health care delivery for which they were developed. Nevertheless, the core aspects of these codes will remain relevant for and helpful to HCPs in humanitarian settings. Humanitarian NGOs may expect HCPs to use their professional codes explicitly or implicitly to guide clinical practice in the field. For example, the Charter of Médecins Sans Frontières states that its 'volunteers promise to honour their professional code of ethics'. ${ }^{4}$ Practicing in ways that are consistent with professional standards may be seen as a critical element of providing competent, ethical care. As such, the professional moral norms of health care disciplines will influence significantly the actions of HCPs in humanitarian work.

These guidance documents and codes (international, humanitarian and professional) can help to orient HCPs towards identifying parameters of ethical practice. However, it will be difficult to derive detailed guidance from them for application in many circumstances. Other tools for ethics analysis are available to support expatriate HCPs as they seek to address the complex situations that arise in humanitarian 
health care practice. As well as the guidelines just described, there are a variety of ethics approaches and moral theories that can be applied to analyse situations of ethical uncertainty. The following subsections contain a brief overview of several normative approaches in relation to ethics decision-making in humanitarian work.

\section{Principle-based accounts}

Humanitarian principles remain the most important aspect of the moral architecture surrounding humanitarian assistance and relief work. Humanitarian principles serve as a scaffold on which the legitimacy of humanitarian action is constructed and a standard by which humanitarian activity is measured (Hilhorst, 2005). ${ }^{5}$ On an organisational level, humanitarian principles have their closest historical connection with the policies and standards of the International Committee of the Red Cross (ICRC). ${ }^{6}$ Humanity, impartiality, neutrality and independence are the fundamental humanitarian principles.

The principle of humanity is the overarching tenet from which the other three fundamental principles flow (Pictet, I985) and entails the 'right to receive humanitarian assistance, and to offer it ...' (IFRC, I994, p. 3).

The principle of impartiality encompasses non-discrimination and proportionality. Non-discrimination emphasises that need alone should be the basis of aid provision and that other distinctions should not factor into aid allocation. Proportionality is the requirement to provide assistance relative to the actual need or degree of suffering.

The principle of independence requires that humanitarian assistance be provided in a way that does not 'further a particular political or religious standpoint' and that NGOs 'endeavour not to act as instruments of government foreign policy' (IFRC, I994, p. 3).

There are differences in how organisations conceive of the principle of neutrality in their operational approaches. One aspect of neutrality that is widely accepted is the notion of non-participation: humanitarian agencies are to refrain from participating in an ongoing conflict, even in an indirect fashion.

Over the past 30 years there has been a broadening of interest in humanitarian principles and increased usage of them by NGOs in mission statements and charters. ${ }^{7}$ The humanitarian principles have been used to develop various inter-agency codes of practice (Weiss, I999). The extent to which HCPs in the field employ humanitarian principles for guidance on resolving practical moral conflicts remains unclear. Hilhorst and Schmiemann (2002) examined the relationship between organisational culture, humanitarian principles and the experiences of practitioners with Médecins Sans Frontières-Holland. They noted that staff members were aware of humanitarian principles but frequently reinterpreted the formal principles in their practice. Humanitarian principles also serve other functions: they act as identity markers for humanitarians and 'interpersonal glue' that provides common purpose and goals for team members (Hilhorst and Schmiemann, 2002).

Formulations of principles that are commonly associated with medical ethics have been employed in evaluating what values are to guide ethically sound health care 
practice in humanitarian settings. These principles are familiar ethical touchstones for many HCPs. Sommers-Flanagan (2007) analyses ethical issues experienced by mental health workers in the context of disasters. To do so she uses the principles of respect for autonomy, nonmaleficience, beneficence, fidelity and justice and brings these principles to bear on the discussion of a series of case studies. She argues that reflecting on principles can stimulate moral imagination and lead those who do so to enact the 'highest moral options available in a given situation' (Sommers-Flanagan, 2007 , p. 200). Principles of medical ethics help to draw attention to key considerations such as avoiding harm, promoting the good of individual patients, respecting and supporting the ability of individuals to make and enact decisions for their lives, and attending to concerns of justice and equity.

Pinto and Upshur (2009) suggest a set of orienting principles to guide medical students during international clinical and research electives. These principles are intended to go beyond clinical decision-making and orient students in a wide range of professional contexts. They should also resonate with other HCPs who volunteer overseas. Pinto and Upshur (2009) encourage trainees to practice in a way that embodies humility, solidarity, introspection and social justice. The inclusion of humility and introspection is a helpful contribution to broadening the set of principles that are considered in health care practice in humanitarian work. For both trainees and HCPs, practicing in a manner that reflects a humble attitude and realistic appreciation of one's capacities and resources may help to prevent potential harm. Likewise, reflection and introspection are essential components of ethical engagement.

As described above, HCPs involved in humanitarian work frequently need to consider the health needs of populations, as well as of individuals. Distinct ethical considerations arise in addressing population and community health needs. The ethical analysis of public health brings considerations of justice to the fore. The rights and needs of individuals are appraised in light of conceptions of the common good. Several formulations of ethics frameworks for public health have been advanced and may provide guidance for HCPs as they incorporate an awareness of population health needs in their clinical practice, and when they participate in public health interventions. Childress et al. (2002) describe five 'justificatory conditions' for evaluating public health interventions: effectiveness; proportionality; necessity; least infringement; and public justification (see Kass (2004) for an alternate framework).

There are important critiques to principlist approaches to decision-making. These critiques have been forcefully articulated in responding to principlism in medical ethics (Clouser and Gert, I990). ${ }^{8}$ Nevertheless, principles are valuable and, when used to inform discussion and reflection, they make an important contribution to ethical deliberation. Principles can act as guideposts and offer a useful vocabulary for examining and discussing ethically complex situations. They signal values and considerations that should be attended to and addressed in evaluating practical moral issues. Moral reasoning is enhanced as principles are considered in relation to relevant contextual features and the moral agency of individuals (Benatar, 2006). 


\section{Consequentialist accounts}

Humanitarian principles have been a central aspect of moral analysis in humanitarian action. Some see a shift, however, in the way that humanitarian actors and commentators assess practical moral issues in emergencies and disasters. In his historical sketch of humanitarianism, Barnett (2005) argues that consequentialist (focusing on outcomes) approaches are gaining ground in humanitarian circles over a traditionally deontological (focusing on duties and obligations) ethic. An increased awareness of the potential unintended, negative consequences of humanitarian assistance has led to an increased focus on outcomes, cost-benefit analyses and efforts to promote accountability (Anderson, I999). There is broad recognition of the potential for unintended harm, and a commitment to evaluate outcomes and develop standards to minimise the negative impacts of assistance. A particular form of moral analysis that attends to the consequences of actions is utilitarianism.

Utilitarianism seeks to maximise aggregate utility for a group or population. When a disaster or complex emergency is in its most acute phase there may be little opportunity to do other than promote a utilitarian approach to decision-making regarding the selection and prioritisation of patients. This will be especially true in situations where need vastly exceeds available resources. An approach that seeks to maximise health for the aggregate of a population may be the most defensible one in such situations. Thus triage will guide HCPs as they seek to identify how to help the greatest number of needy individuals in light of the severity of injuries and other medical criteria.

\section{Virtue ethics and role models}

Slim (I997) finishes his thoughtful and engaging discussion of relief agencies, moral dilemmas and moral responsibility with an exploration of moral role models in humanitarian work. He observes that many people are guided in their moral choices by reference to what particularly virtuous individuals might do in a similar situation. This focus draws on the rich literature on virtue ethics that stretches back to Aristotle (I980). Slim argues that this approach is a potential, and largely untapped, source of moral guidance for humanitarian practitioners. He notes that traditionally vaunted figures of humanitarianism such as Florence Nightingale or Henri Dunant are not the best role models to serve this purpose. Instead, he encourages humanitarian practitioners to seek moral role models from among the local population. Slim (I997, p. 256) asserts that '[t] he moral role models which agencies need are likely to be right there in front of them in their clinics, their staff, their partners and in the long queues for their food rations'. HCPs also may benefit from considering what a respected colleague or mentor would do if faced with the situation at hand. Such acts of imagination that aim to identify how a virtuous and skilful practitioner would respond in similar circumstances can provide new perspectives for assessing morally complex situations. A virtue ethics approach also may be in greater harmony with local frameworks and values in some non-Western contexts than rightsor principle-based accounts (Widdows, 2007). 


\section{Human rights}

Human rights are a powerful tool for analysing ethical issues in humanitarian settings. Human rights have a particular value in considering the role of NGOs in protecting vulnerable individuals in a context of war or disaster, as well as drawing attention to the responsibilities of states and other actors. The mandate of some NGOs is directly linked to advocacy and the promotion of human rights. Rights that are relevant to health care practice in humanitarian work include both positive and negative rights. Positive rights include entitlements 'to' certain things, such as the right to life or the right to health. At the broadest level all humanitarian action can be seen as a response to the right to a minimum standard of health for individuals affected by a humanitarian emergency or disaster (Gunn, 2003). Although the historical legal justification for humanitarian action has been grounded in international humanitarian law, there is increasing recognition that human rights also provide a robust foundation for humanitarian relief work (Leaning, I999; Darcy 2004). Human rights offer guidance in providing safeguards for the protection of individuals caught up in humanitarian crises. In some contexts, though, humanitarian practitioners and agencies may struggle to balance the need to challenge injustices while maintaining access to affected populations (Leader, I998). An important impact of considering rights in regard to humanitarian action is that such an approach (in contrast to a focus on charity) emphasises the agency of the holders of rights (Alkire and Chen, 2004). This point also may be true on a larger scale; a focus on the right of affected populations to receive care can influence how beneficiaries are perceived by different actors. Beneficiaries are characterised not as objects but as subjects (Rieff, 2002).

\section{Questions to orient ethics decision-making in humanitarian work}

Ethical theories and normative frameworks can provide assistance as HCPs seek to respond with integrity and compassion to individuals and communities in situations of great need. These structures offer resources that nourish reflection and deliberation; however, there are no simple or mechanistic solutions to complex ethical issues. HCPs will still struggle to apply ethics frameworks in the uncertain and unpredictable reality of humanitarian health care practice.

The following section proposes a set of questions to support reflection and discussion of ethically-fraught issues in health care practice in humanitarian settings. ${ }^{9}$ As HCPs in humanitarian work encounter ethically complex issues a number of lines of reflection will assist them in gathering needed information, critically assessing the situation and its context, and involving appropriate stakeholders. This process draws attention to ethics resources that may help to illuminate the ethical issue. Different ethical theories and approaches provide lenses through which a particular issue can be examined and can reveal important facets of the issue that should be considered (Sherwin, I999). The ability to work through such questions is dependent 
on resources of time, energy, communication and staffing. HCPs, in teams or individually, may find the following lines of reflection useful as they seek to evaluate an issue that has important ethical dimensions. In using this tool, HCPs may omit questions, consider questions out of order, and move back and forth among the questions. The questions are not intended to be discrete or exclusively unidirectional.

\section{Identify/clarify the ethical issue}

What is the issue that we are experiencing?

What is at stake and for whom?

How is this issue experienced/understood from different perspectives?

\section{Data gathering and attention to context}

a) Who can contribute to helping us understand this issue better? Have we involved these individuals? When national HCPs are not part of the treatment team, discussion with local health workers or community representatives will be an important step. ${ }^{\text {Io }}$ In addition, there may be colleagues within the NGO, perhaps in a neighbouring project or at headquarters, who can provide insight on the issue.

b) Have we identified and understood (as best as we can) relevant contextual features? In particular, it will be important to question how cultural frameworks and personal (and collective) histories affect how the issue is understood.

c) How do organisational features influence the issue? This analysis could include exploration of the impact of staff turnover, organisational culture, clarity of programme and organisational objectives, and structures of accountability and responsibility.

d) How do local legal and professional parameters of practice relate to the issue?

e) What is the impact of the professional and social norms of our home countries on how we understand this issue?

f) What impact do personal biases, goals and values have on our understanding of the situation? How do these perspectives relate to the expectations, views and values of the local community and others involved in this situation?

g) What is the impact of this issue on collaboration between different actors (individual and collective) and on trust in these relationships?

h) How are imbalances of power relevant to the issue? Have asymmetries of power been ignored or taken advantage of? Are there opportunities to enhance and promote (individual and collective) decision-making and contribute to the development of local capacity?

i) Are people being treated unequally without a sound rationale for doing so?

\section{Review of ethical issue}

Do the answers to these questions reveal new aspects of the ethical issue?

Does this lead us to reformulate or redefine the issue?

\section{Exploration of ethics resources}

What ethics resources and approaches can assist us in evaluating this issue? What values and norms ought to inform our decision-making? The various ethical frameworks and resources discussed in this paper can be considered. 


\section{Evaluation of possible options and selection of the best option}

What actions or options are possible in this situation and what ethical norms support each option? What consequences might result from each option? How do options relate to professional obligations and duties? Can consequences, values and obligations be reconciled? What might be lost if particular options are selected? Options supported by less important rationales should be set aside or given low priority. Potential positive and negative consequences should be weighed, and the 'best' option selected. ${ }^{\text {II }}$

\section{Implementation and follow-up}

a) What steps are required to implement the selected option?

Following implementation of the selected option the team should consider:

b) Did the anticipated outcome actually take place? Are there things that we missed or did not account for in our analysis? What can we learn from this situation?

c) Is there a need for retrospective debriefing for those involved or affected?

The goal of these questions is to encourage a deliberative process where appropriate persons are included in the discussion, relevant contextual features are accounted for, the influence of values and power is considered, and the application potential of different ethics resources is evaluated. The proposed questions can lead HCPs towards a more comprehensive understanding of the issue, and assist them in identifying and enacting a well-considered and ethically defensible decision in a particular situation. This set of questions should not be seen as a recipe for resolving ethics issues. Rather it is a tool to promote and guide reflection, discussion and ethical action.

\section{Conclusion}

The ethics of humanitarian action have received considerable attention from academics, policymakers and practitioners. This has led to important discussion of values, practices and structures of humanitarian assistance, and resulted in the development of standards of practice, accountability initiatives, and codes of conduct for humanitarian assistance work. Ethical issues experienced by HCPs in humanitarian settings, and ethical frameworks for appraising this work, have yet to attract the same amount of analysis and debate. Developing and refining ethics resources and vocabulary for health care practice in humanitarian work will help HCPs to reflect, deliberate, and provide ethically defensible care to patients and populations. Although there are common features in the moral landscape of humanitarian health care practice, there will be many situations that are impossible to anticipate. HCPs apply 'theoretical knowledge, common sense, improvisation, or trial-and-error' (Bjerneld et al., 2004, p. I05) approaches to work through challenging issues in humanitarian work. This paper contributes to the development of theoretical knowledge that can inform ethical analysis in humanitarian settings. It elaborates a set of questions to help guide HCPs as they assess ethical issues. This process can assist HCPs in establishing their moral bearings in situations of ethical complexity and uncertainty. It 
is hoped that this analysis will contribute to, and stimulate, discussion of ethical issues in humanitarian health care practice.

\section{Acknowledgements}

An earlier version of this paper was included in the author's doctoral dissertation at the Division of Experimental Medicine, McGill University, Montreal, Canada. Thanks are extended to dissertation supervisor, Leigh Turner, and to the members of the thesis committee, Franco Carnevale, Carolyn Ells and Steven Jordan, for their assistance and support. Thanks too to James Anderson, Hillel Braude, Avi Craimer and Lisa Schwartz, as well as to the anonymous reviewers for their helpful comments on this manuscript. The author's research is supported by a post-doctoral fellowship from the Canadian Institutes of Health Research.

\section{Correspondence}

Matthew R. Hunt, Post-Doctoral Fellow, Centre for Research on Ethics (CRÉUM), University of Montreal, C.P. 6I28, succ. Centre-ville, Montreal, QC, $\mathrm{H}_{3} \mathrm{C}$ 3J7, Canada. Telephone: +5I4 343-6II (ext. I354); fax: +5I4 343-7899.

E-mail: Imatthew.hunt@gmail.com

\section{Endnotes}

I This paper focuses on expatriate HCPs who work with international non-governmental organisations (NGOs). Aspects of this discussion also are applicable to national HCPs who work with aid agencies. However, some elements of the analysis presented here relate specifically to HCPs who travel from their home nation to work in settings of disaster or conflict (for example, issues related to professional oversight and ethical requirements of the licensing body in one's home country). The author also acknowledges that there are important differences between the provision of health care in settings of natural disaster and in situations of acute or chronic political instability or armed conflict. Given the scope and goals of this paper, this author believes there are sufficient similarities between such settings to justify discussing them together.

2 To develop this analysis the author draws on published empirical studies, normative commentaries, as well as his programme of qualitative research exploring ethical issues for HCPs involved in humanitarian assistance and development work. The author has participated in three qualitative studies with HCPs involved in global health initiatives (Hunt, 2008, 2009; Schwartz et al., 2010). These studies collectively included in-depth interviews with 45 Canadian HCPs.

3 Ideas of charity and equity may conflict and may motivate divergent responses to situations of need.

4 See http://www.msf.ca/about-msf/msf-charter/.

5 The development of the fundamental humanitarian principles is closely linked to international humanitarian law. This body of law (particularly the Geneva Conventions and their Additional Protocols) delineates responsibilities of states in allowing access to affected populations for the purposes of providing humanitarian relief (Pictet, I985). International humanitarian law describes 
particular conditions relating to when and how states are to permit such access. The fundamental humanitarian principles function as broad descriptions of these conditions (Mackintosh, 200o).

6 The seven principles of the ICRC are humanity, impartiality, neutrality, independence, voluntary service, unity, and universality (Pictet, I979).

7 A variety of factors have contributed to the increased interest in humanitarian principles, including proliferation and diversification of aid agencies, changes in the nature of conflicts, and greater uncertainty regarding the ethics of humanitarian action (Weiss, I999; Hilhorst and Schmiemann, 2002).

8 Briefly, critics of principlist approaches highlight the following: specific principles may conflict in a given situation and there is rarely guidance on how to prioritise from among principles; principles may not provide clear guidance for decision-making in real-world situations; it is difficult to justify which principles to include and which to exclude; and since principles are linked to particular moral traditions, privileging one set of mid-level principles can tacitly elevate a particular moral vision above others.

9 The proposed set of questions draws on existing models (Purtillo, I993, pp. 49-57; MacDonald, 200I) with the goal of elaborating guidance for ethics decision-making that is adapted to the specific context of health care practice in humanitarian work. These questions do not focus specifically on issues in clinical care but encompass the organisation and structure of health care in humanitarian projects. Development of the questions was informed by the qualitative research that the author conducted with HCPs engaged in humanitarian and development work. These questions have been not been piloted or tested with HCPs. The author intends to evaluate the usefulness of this approach through focus groups of experienced HCPs.

Io When considering seeking outside input, considerations related to confidentiality should be carefully evaluated.

II Alternately, a cluster of options could be identified that together address the issue.

\section{References}

Alkire, S. and L. Chen (2004) 'Global health and moral values'. The Lancet. 364(9439). pp. Io69-I074. Anderson, M.B. (I999) Do No Harm: How Aid Can Support Peace-Or War. Lynne Rienner, Boulder, CO.

Anderson, M.B. (2000) 'Aid: a mixed blessing'. Development in Practice. Io(3-4). pp. 495-500.

Aristotle (I980) The Nicomachean Ethics. Translated by D. Ross; revised by J. L. Ackrill and J. O. Urmson. Oxford University Press, Oxford.

Banatvala, N. and A.B. Zwi (2000) 'Public health and humanitarian interventions: developing the evidence base'. British Medical Journal. 32I(7253). pp. IOI-IO5.

Barnett, M. (2005) 'Humanitarianism transformed'. Perspectives on Politics. 3(4). pp. 723-740.

Benatar, S.R. (2006) 'Facing ethical challenges in rolling out antiretroviral treatment in resourcepoor countries: comment on “They call it 'patient selection' in Khayeltisha”. Cambridge Quarterly of Healthcare Ethics. I5 (3). pp. 322-330

Bhan, A. (2005) 'Should health professionals allow reporters inside hospitals and clinics at times of natural disasters?' PLOS Medicine. 2 (6). pp. O47I-0473.

Bjerneld, M., G. Lindmark, P. Diskett and M.J. Garrett (2004) 'Perceptions of work in humanitarian assistance: interviews with returning Swedish health professionals'. Disaster Management and Response. 2(4). pp. IOI-IO8.

Brennan, R.J. and R. Nandy (200I) 'Complex humanitarian emergencies: a major global health challenge'. Emergency Medicine. I3 (2). pp. I47-I56.

Childress, J.F. et al. (2002) 'Public health ethics: mapping the terrain'. Journal of Law, Medicine and Ethics. $30(2)$. pp. I70-I78. 
Clouser, K. and B. Gert (I990) 'A critique of principlism'. Journal of Medical Philosophy. I5 (2). pp. 219-236. Crigger, N.J. and L. Holcomb (2007) 'Practical strategies for providing culturally sensitive, ethical care in developing nations'. Journal of Transcultural Nursing. I8(I). pp. 70-76.

Darcy, J. (2004) Human Rights and Humanitarian Action: A review of the Issues. Humanitarian Policy Group Background Paper. Overseas Development Institute, London.

de Waal, A. (1998) Famine Crimes: Politics and the Disaster Relief Industry in Africa. Indiana University Press, Bloomington, IN.

Frangonikolopoulus, C.A. (2005) 'Non-governmental organizations and humanitarian action: the need for a viable change of praxis and ethos'. Global Society. I9(I). pp. 50-72.

Fuller, L. (2006) 'Justified commitments? Considering resource allocation decisions and fairness in Medecins sans Frontieres-Holland'. Developing World Bioethics. 6(2). pp. 59-70.

Gunn, S.W.A. (2003) 'The right to health of disaster victims'. Disaster Management and Prevention. I2 (I). pp. 48-5I.

Hakewill, P. (I997) 'Doctoring beyond frontiers'. Medical Journal of Australia. I67(II-I2). pp. 6I8-62 I.

Hilhorst, D. (2005) 'Dead letter or living document? Ten years of the Code of Conduct for disaster relief'. Disasters. 29(4). pp. 35I-369.

Hilhorst, D. and N. Schmiemann (2002) 'Humanitarian principles and organizational culture: everyday practice in Médecins Sans Frontières-Holland'. Development in Practice. I2 (3-4). pp. 490-500.

Hoffmaster, B. (I994) 'The forms and limits of medical ethics'. Social Science and Medicine. 39(9). pp. II55-II64.

Horton, K. (2008) 'Transnational medical aid and the wrongdoing of others'. Public Health Ethics. I(2). pp. I7I-I79.

Humanitarian Accountability Partnership-International (2007) HAP 2007 Standard in Humanitarian Accountability and Quality Management. Humanitarian Accountability Partnership-International, Geneva. http://www.hapinternational.org/pool/files/hap-2007-standard(I).pdf (accessed on 2 I December 20IO).

Hunt, M.R. (2008) 'Ethics beyond borders: how health professionals experience ethics in humanitarian assistance and development work'. Developing World Bioethics. 8(2). pp. 59-69.

Hunt, M.R. (2009) 'Moral experience of Canadian healthcare professionals in humanitarian work'. Prehospital and Disaster Medicine. 24(6). pp. 5I8-524.

Hurst, S.A., N. Mezger and A. Mauron (2009) 'Allocating resources in humanitarian medicine'. Public Health Ethics. 2(I). pp. 89-99.

ICN (International Council of Nurses) (2006) Code of Ethics for Nurses. Revised (first adopted in I953). ICN, Geneva.

IFRC (International Federation of Red Cross and Red Crescent Societies) (I994) The Code of Conduct for the International Red Cross and Red Crescent Movement and NGOs in Disaster Relief. IFRC, Geneva.

IFRC (2003) World Disasters Report 2003: Focus on ethics in aid. IFRC, Geneva.

Kass, N. (2004) 'Public health ethics: from foundations to frameworks to justice and global public health'. Journal of Law, Medicine and Ethics. $32(2)$. pp. 232-242.

Kleinman, A. (1980) Patients and Healers in the Context of Culture. University of California Press, Berkeley, CA.

Leader, N. (I998) 'Proliferating principles; or how to sup with the devil without getting eaten'. Disasters. $22(4)$. pp. 288-308.

Leaning, J. (I999) 'Medicine and international humanitarian law'. British Medical Journal. 3 I9 (7207). pp. 393-394.

Loquercio, D., M. Hammersley and B. Emmens (2006) Understanding and Addressing Staff Turnover in Humanitarian Agencies. Humanitarian Practice Network Paper. 55. Overseas Development Institute, London. http://www.odihpn.org/documents/networkpapero55.pdf(accessed on 2I December 20IO). 
MacDonald, M. (200I) A Framework for Ethical Decision-Making: Version 6.o Ethics Shareware. http:// www.ethics.ubc.ca/upload/A\%20Framework\%20for\%20Ethical\%2oDecision-Making.pdf (accessed on I6 December 20I0).

Mackintosh, K. (2000) The Principles of Humanitarian Action in International Humanitarian Law. Study 4 in 'The politics of principle: the principles of humanitarian action in practice'. Humanitarian Policy Group Report. 5 (March). Overseas Development Institute, London. http://www.odi.org. $\mathrm{uk} /$ resources/download/249.pdf (accessed on I6 December 20I0).

Mann, J. (I997). 'Medicine and public health, ethics and human rights'. The Hastings Center Report. 27(3). pp. 6-I3.

Michael, M. and A.B. Zwi (2002) 'Oceans of need in the desert: ethical issues identified while researching humanitarian agency response in Afghanistan'. Developing World Bioethics. 2(2). pp. I09-I30.

Moore, J. (ed.) (1998) Hard Choices: Moral Dilemmas in Humanitarian Intervention. Rowman and Littlefield Publishers, Lanham, MD.

Parfitt, B. (I999) 'Working across cultures: a model for practice in developing countries'. International Journal of Nursing Studies. 36(5). pp. 371-378.

People in Aid (2003) Code of Good Practice in the Management and Support of Aid Personnel. http://www. peopleinaid.org/pool/files/code/Code\%20of\%2oGood\%2oPractice\%20-\%2oFinal\%202007.pdf (accessed on 2I December 20I0).

Pictet, J. (I979) The Fundamental Principles of the Red Cross: Commentary. http://www.icrc.org/eng/ resources/documents/misc/fundamental-principles-commentary-oroI79.htm (accessed on I6 December 2010).

Pictet, J. (I985) Development and Principles of International Humanitarian Law. Martinus Nijhoff Publishers, Dordrecht.

Pinto, A. and R. Upshur (2009) 'Global health ethics for students'. Developing World Bioethics. 9(I). pp. I-IO.

Purtillo, R. (I993) Ethical dimensions in the health professions. W.B. Saunders, Philadelphia, PA.

Rieff, D. (2002) A Bed for the Night: Humanitarianism in Crisis. Simon and Schuster, New York, NY.

Schwartz, L. et al. (2010) 'Ethics in conditions of disaster and deprivation: learning from health workers' narratives'. American Journal of Bioethics - Primary Research. I (3). pp. 45-54.

Sherwin, S. (I999) 'Foundations, frameworks, lenses: the role of theories in bioethics'. Bioethics. I3 $3(3-4) \cdot$ pp. I98-205.

Shweder, R., N. Much, M. Mahpatra and L. Park (I997) 'The "Big three" of morality (autonomy, community, divinity) and the "big three" explanations of suffering'. In A. Brandt and P. Rozin (eds.) Morality and Health. Routledge, New York, NY. pp. I I9-I69.

Slim, H. (I997) 'Doing the right thing: relief agencies, moral dilemmas and moral responsibility in political emergencies and war'. Disasters. 2 I (3). pp. 244-257.

Sommers-Flanagan, R. (2007) 'Ethical considerations in crisis and humanitarian interventions'. Ethics and Behaviour. I7(2). pp. I87-202.

Sphere Project (2004) The Humanitarian Charter and Minimum Standards in Disaster Response. http://www. sphereproject.org/content/view/27/84/ (accessed on 2I December 20IO).

Terry, F. (2002) Condemned to Repeat? The Paradox of Humanitarian Action. Cornell University Press, Ithaca, NY.

Tong, R. (200I) 'Towards a feminist global bioethics: addressing women's health concerns worldwide'. Health Care Analysis. 9(2). pp. 229-246.

Weiss, T.G. (I999) 'Principles, politics and humanitarian action'. Ethics and international Affairs. I3(I). pp. I-22.

Wheeler, V. and A. Harmer (eds.) (2006) Resetting the Rules of Engagement: Trends and Issues in MilitaryHumanitarian Relations. Humanitarian Policy Group Research Briefing. 2I (March). Overseas Development Institute, London. http://www.odi.org.uk/resources/download/263.pdf (accessed on 16 December 20I0). 
Widdows, H. (2007) 'Is global bioethics moral neo-colonialism? An investigation of the issue in the context of bioethics'. Bioethics. 2I(6). pp. 305-3I5.

WMA (World Medical Association) (2006a) International Code of Medical Ethics. Adopted in London, United Kingdom, in October I949, and amended in Sydney, Australia, in August I968, in Venice, Italy, in October I983, and in, Pilanesberg, South Africa, October 2006.

WMA (2006b) Statement on Medical Ethics in the Event of Disasters. Adopted in Stockholm, Sweden, in September I994, and revised in Pilanesberg, South Africa, in October 2006. 